University of Nebraska - Lincoln

DigitalCommons@University of Nebraska - Lincoln

2009

\title{
Self-Assembly of Magnetic Ni Nanoparticles into 1D Arrays with Antiferromagnetic Order
}

\author{
Valery Bliznyuk \\ Western Michigan University \\ Srikanth Singamaneni \\ Western Michigan University, singamaneni@wustl.edu \\ Sarbeswar Sahoo \\ University of Nebraska, Lincoln, sarbeswar@gmail.com \\ Srinivas Polisetty \\ University of Nebraska, Lincoln, polisetty.srinivas@gmail.com \\ $\mathrm{Xi} \mathrm{He}$ \\ University of Nebraska, Lincoln \\ See next page for additional authors
}

Follow this and additional works at: https://digitalcommons.unl.edu/physicsbinek

Part of the Physics Commons

Bliznyuk, Valery; Singamaneni, Srikanth; Sahoo, Sarbeswar; Polisetty, Srinivas; He, Xi; and Binek, Christian, "Self-Assembly of Magnetic Ni Nanoparticles into 1D Arrays with Antiferromagnetic Order" (2009). Christian Binek Publications. 63.

https://digitalcommons.unl.edu/physicsbinek/63

This Article is brought to you for free and open access by the Research Papers in Physics and Astronomy at DigitalCommons@University of Nebraska - Lincoln. It has been accepted for inclusion in Christian Binek Publications by an authorized administrator of DigitalCommons@University of Nebraska - Lincoln. 


\section{Authors}

Valery Bliznyuk, Srikanth Singamaneni, Sarbeswar Sahoo, Srinivas Polisetty, Xi He, and Christian Binek 


\title{
Self-assembly of magnetic Ni nanoparticles into 1D arrays with antiferromagnetic order
}

\author{
V. Bliznyuk, ${ }^{1}$ S. Singamaneni, ${ }^{1}$ S. Sahoo, ${ }^{2}$ S. Polisetty, ${ }^{2}$ Xi He, ${ }^{2}$ and C. Binek ${ }^{2}$ \\ 1 College of Engineering and Applied Sciences, Western Michigan University, Kalamazoo, MI 49008, \\ USA \\ 2 Department of Physics and Astronomy and the Nebraska Center for Materials and Nanoscience, \\ University of Nebraska-Lincoln, Lincoln, NE 68588, USA
}

Corresponding authors - V. Bliznyuk, valery.bliznyuk@wmich.edu and C. Binek, cbinek2@unl.edu

\begin{abstract}
In this paper, we report on the magnetic properties of isolated nanoparticles and interacting nanochains formed by the self-assembly of Ni nanoparticles. The magnetic properties were studied using superconducting quantum interference device (SQUID) magnetometry and magnetic force microscopy (MFM). We demonstrate that single-domain Ni nanoparticles spontaneously form one-dimensional (1D) chains under the influence of an external magnetic field. Furthermore, such magnetic field-driven self-assembly in conjunction with surface templating produces regular arrays of 1D nanochains with antiferromagnetic intra-chain order. The antiferromagnetic order, which is in striking contrast to what is found for non-interacting nanoparticle assemblies within the chains, can be evidenced from MFM and SQUID measurements.
\end{abstract}

\section{Introduction}

While magnetism at different length scales (planetary, macro, and nano) has intrigued-and continues to intrigue-humans for a long time, it required quantum statistics to recognize that magnetism is largely a nanoscale phenomenon [1]. Nanostructured magnetic materials have the potential to revolutionize current data storage technologies [2, 3], magnetoelectronics [4-6], and biotechnology (cell separation, immobilized enzymes, protein separations, hyperthermia, and target drugs) [7, 8]. As the demand for higher density in recording media increases, a fine control of the shape and arrangement of magnetic domains becomes more important in order to not only enhance densities of magnetic bits but also to have a fine control over "cross-talk" between magnetic segments. Creation of single- domain magnetic arrays with individual domain size below $50 \mathrm{~nm}$, for instance, could lead to storage densities of over 1 Tbit/in² [9]. The so-called "spintronic devices" which are designed to exploit the spin of the electrons as opposed to the mere charge of the electrons in conventional electronic systems are being intensely investigated [10-12].

From a basic scientific point of view, nano-patterned magnetic materials are also of great interest in the sense that the role of low dimensionality becomes a fundamental issue in the magnetic properties of such materials. For example, as the size of the individual magnetic nanoparticle reduces, it approaches the superparamagnetic limit at room temperature $[13,14]$. In the superparamagnetic state, an inter-particle ordered state is not established within the time frame of static magnetic measurements, $t=100 \mathrm{~s}$ [1518]. Each moment thermally fluctuates between two minima with oppositely directed magnetization following an Arrhenius type relaxation time $t=t_{0} \exp \left(K V / k_{\mathrm{B}} T\right)$, where $K$ is the anisotropy constant of a particle of volume $V$ at temperature $T$, and $t_{0}$ is a microscopic limiting relaxation time usually $\sim 10^{-9} \mathrm{~s}$. The overall magnetic moment is therefore equal to zero and the material does not possess macroscopic magnetization. 

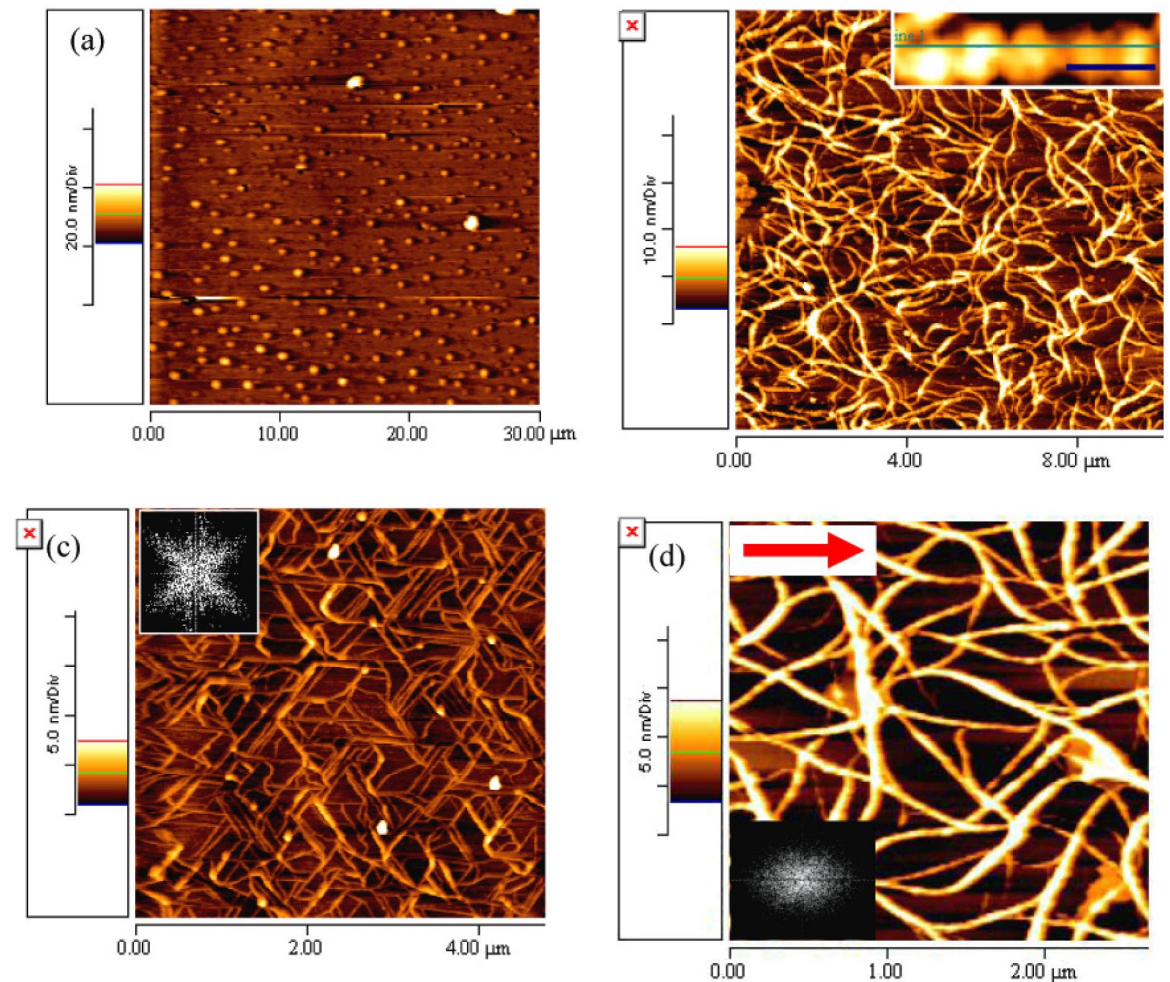

Figure 1. AFM images of (a) Ni nanoparticles cast on Si substrate, (b) Ni nanochains formed by self-organized nanoparticles in the presence of magnetic field (the inset shows higher magnification of a single nanochain, scale bar of $200 \mathrm{~nm}$ ), (c) Ni nanochains assembled on HOPG showing the preferential alignment of the chains along the step edges (inset shows the FFT of the AFM image showing the hexagonal symmetry), (d) Nanochains assembled on Si substrate tilted at 30. during solvent evaporation (arrow shows the direction of the flow field during solvent evaporation and inset shows the FFT of the image with preferential alignment).

Various non-lithographic techniques have been demonstrated for the synthesis of Ni nanoparticles which include-but are not limited to-high temperature organometallic decomposition, electrochemical reduction, and chemical reduction [19-26]. The above methods have been intensely investigated and optimized to achieve a precise control over the size and shape of the nanoparticles [27]. In particular, the chemical routes for the fabrication of nanoparticles involve (1) use of emulsions as nanoreactors to nucleate nanoparticles and (2) nucleation of nanoparticles in high temperature organic solvents. The first method involves the preparation of monodisperse metallic and metal oxide nanoparticles using micelles, which provides fine control over the particle size. The second method involves the nucleation and growth of nanoparticles in an organic solvent. The technique results in magnetic nanoparticles with good crystallinity and a relatively monodisperse, controlled size distribution. With the exception of a few instances, a comprehensive characterization of the magnetic properties (e.g. magnetic behavior, nature of interaction) of the so formed nanoparticles as a function of the size, shape, and assembly is clearly lacking.

The assembly of nano-magnetic structures into macroscopic domains by self-organization has attracted significant attention and offers considerable advantages over the conventional lithographic processes [28-33]. Biological systems provide excellent examples of self-assembly of mag- netic nanoparticles enabling unique sense of direction with respect to geo-magnetic field. Chains of 40-100 nm magnetite nanoparticles were observed in magnetotactic bacteria useful for their vertical orientation. The chain-like assembly of the particles causes a permanent magnetic dipole, which is critical for the orientation [34].

Understanding and controlling the properties of the magnetic nanostructures is paramount for an efficient application. We have recently demonstrated a rather simple and efficient way of fabrication of colloidal solution of Ni nanoparticles and their self-assembly into arrays of weakly interacting nanoparticles or strongly coupled nanochains [35]. Here we primarily focus on the correlation between the structure and magnetism of the self-organized Ni nanochains.

\section{Results and discussion}

Colloidal solution of $\mathrm{Ni}$ nanoparticles was prepared by a two stage procedure (vacuum deposition of thin films followed by ultrasonic treatment in chloroform) described in our previous publication [35]. Solution of Ni nanoparticles was then cast on the silicon substrate and the size distribution of the particles was estimated using atomic force microscopy (AFM) imaging (Autoprobe CP microscope, in noncontact mode or magnetic force microscopy mode with magnetized Co coated tips as discussed below). Figure 1(a) 
depicts an AFM image of Ni nanoparticles cast on silicon substrate showing uniform distribution of nanoparticles on the substrate. A narrow distribution of the particle size was observed from AFM imaging with a mean diameter of 9.5 $\mathrm{nm}$. The particles were found to be randomly distributed with no specific alignment or aggregation.

Self-organization of magnetic nanoparticles into chains was achieved by performing the solvent evaporation in the presence of external magnetic field. Ni nanoparticles were casted in the presence of a magnetic field of strength $250 \pm$ $10 \mathrm{G}$ as measured by Bell 620 Gaussmeter. Casting the Ni nanoparticle solution in the presence of the magnetic field and subsequent solvent evaporation resulted in the spontaneous assembly of the nanoparticles into interconnected network of nanochains. Once the solution is cast on the substrate and subjected to magnetic field, the assembly of the nanoparticles into nanochains possibly occurs in the solution itself. However, the assembly of the flexible nanochains on the substrate is dictated by the combination of the hydrodynamic forces on the nanochains and surface tension. It is worth noting that the absence of magnetic field during the casting and solvent evaporation resulted in isolated particles randomly distributed on the surface with no signs of aggregation. When the solvent evaporation was done in the presence of magnetic field, a size discriminative self-assembly of the particles into chains was observed. AFM image in Figure 1(b) shows the branched network of chains of nanoparticles with an average length of the chain between 2 and $3 \mu \mathrm{m}$. A careful observation of the image also shows that all the elongated wire-like structures are actually granular in nature comprising of the individual nanoparticles (inset of Figure 1(b)). Moreover, we noticed that there are very few isolated particles (less than 10\%), which remain isolated without falling into chains. The isolated smaller nanoparticles, which are not involved in the process of chain formation, reveal superparamagnetic effect.

Spontaneous organization under magnetic field was combined with template-directed as well as flow (magnetic) field-directed organization in order to induce anisotropic orientation into the nanochains. The assembly of the nanoparticles was carried out on a highly oriented pyrolytic graphite (HOPG) template with atomic step edges, which can effectively act as physical confinements directing the assembly of nanochains. HOPG was previously employed as a template for the electrodeposition of a variety of nanowires for gas sensor applications [36-38]. Figure 1(c) shows a typical AFM micrograph of the nickel nanoparticles self-assembled on the surface of HOPG forming chains. It can be observed that the chains are rather straight and continuous compared to those formed on silicon substrate. It can be inferred that the nanochains follow the atomic dislocations or the so-called atomic step edges on the surface of HOPG formed during cleaving process. HOPG with atomic steps typically $0.3-2 \mathrm{~nm}$ in height acts as an excellent template resulting in a directed self-assembly of the nanoparticles. In a different experiment, flow induced alignment of the particles in conjunction with magnetic field by casting the solution on tilted substrate resulted in anisotropic nanochains.

It has been previously demonstrated that various onedimensional structures such as carbon nanotubes and DNA

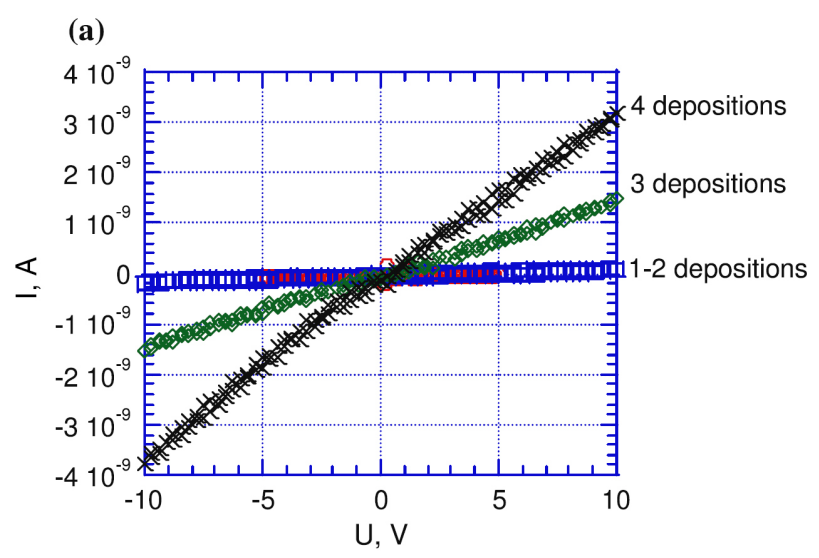

(b)

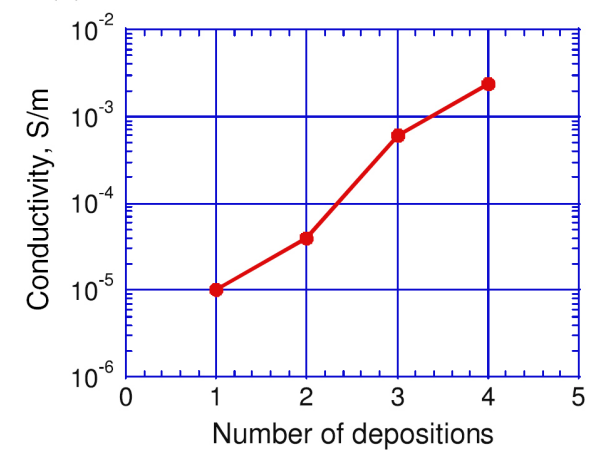

Figure 2. (a) $I-V$ characteristics of the nanochains after four subsequent depositions formed under external magnetic field depicting the percolation network formed by the nanochains. (b) Plot depicting the electrical conductivity of the Ni nanochain network for various number deposition of the nanoparticles.

can be aligned by the receding contact line during drying process [39-41]. Figure 1(d) presents the AFM image of such a sample prepared with the $\mathrm{Ni}$ nanoparticle solution flowing in the direction of magnetic field on a silicon substrate inclined at nearly $30^{\circ}$ from horizontal position. The inset shows the FFT of the AFM image depicting small degree of preferential orientation of the nanochains (as indicated by an arrow). One can speculate that when the nanoparticle solution is cast on the tilted substrate, the liquid-solid-air contact line is moving slowly down the surface of the substrate due to the solvent evaporation. This process results in the accumulation of highly concentrated solution of the nanochains in the vicinity of the receding contact line, thus leaving dense and reasonably oriented nanochains on the surface.

While the AFM images revealed a highly interpenetrating network of Ni nanochains due to the external magnetic field, electrical conductivity provides evidence for the macroscopic percolation network of the Ni nanochains. The electrical properties of the network of nanochains were tested by the current-voltage $(I-V)$ characteristics (Keithley 2400 electrometer operated with PC Labview software). For this purpose, patterned silver microelectrodes across the $\mathrm{Ni}$ nanochains were fabricated by thermal evaporation of silver. Figure 2(a) depicts the $I-V$ characteristics of the $\mathrm{Ni}$ nanochains after 4 subsequent depositions. As expected for a continuous network of nanochains, the ohmic 

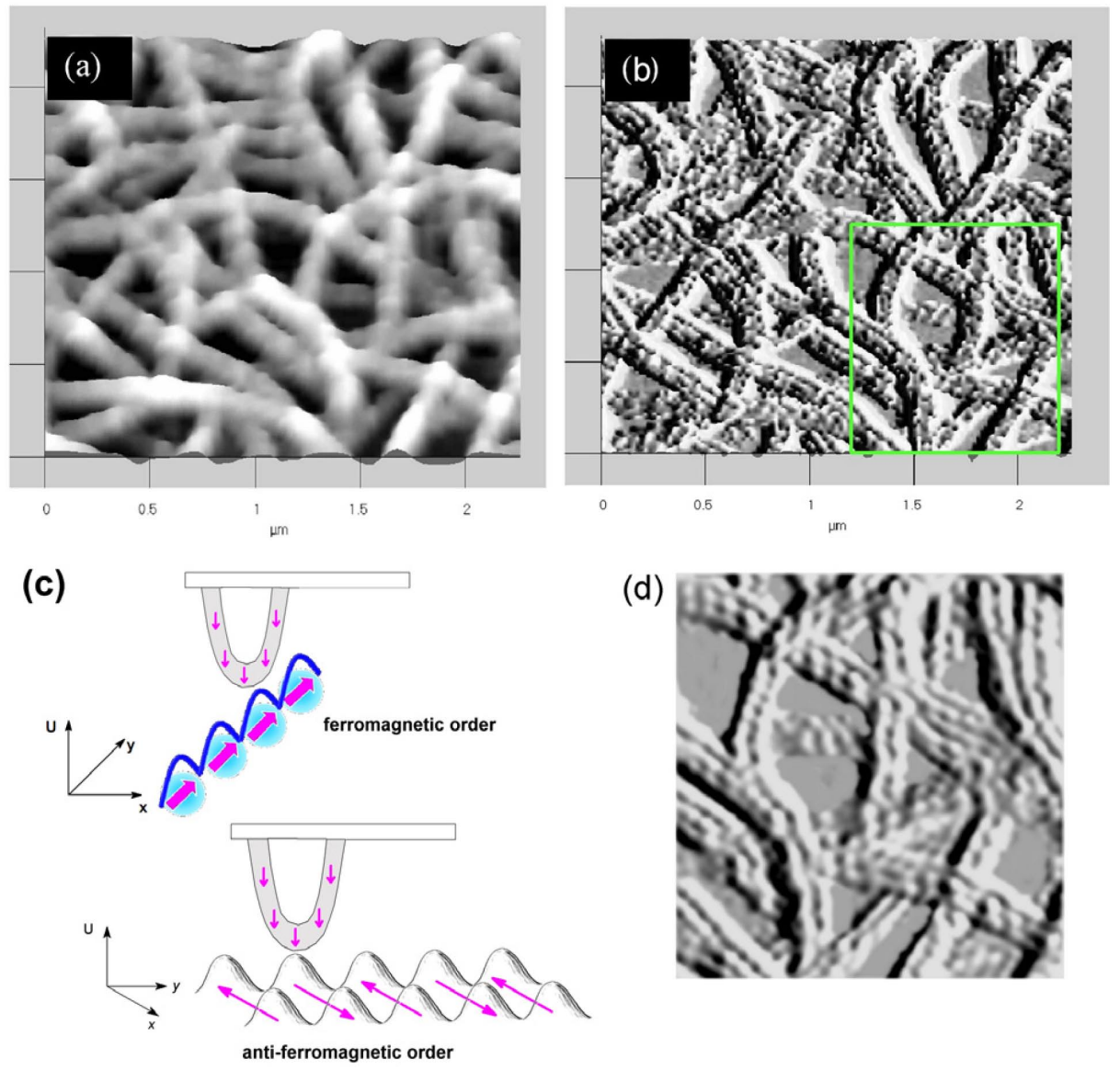

Figure 3. (a) AFM topography and (b) MFM images of the same region of Ni nanochains on silicon substrate. (c) Schematic of the suggested mechanism for the observed MFM contrast with antiferromagnetic order of magnetic Ni nanoparticles along the chain. Arrows show orientation of magnetic moments of individual nanoparticles and that of the AFM tip coated with Co and magnetized in the direction along the tip. $U$ represents repulsive interaction of the AFM tip with the sample; $y$ is direction along the chain; and $x$ across the chain. In cases of ferromagnetic arrangement of the magnetic moments within the chain (top portion), MFM signal roughly resembles the topography; while in cases of antiferromagnetic order (bottom portion), the chain appears as a two strand line of bumps due to the possible interaction of the tip with the magnetic moment of the closest nanoparticle, as well as with the magnetic moments of neighboring nanoparticles. (d) Zoom-in of the MFM image followed by high frequency Fourier filtration, emphasizing the existence of magnetic field variation along the chains corresponding to antiparallel arrangement of magnetic moments as shown in portion (c).

conductivity (i.e., the slope of $I-V$ curve) monotonically increases with the number of depositions. Figure 2(b) shows the plot of the electrical conductivity of the Ni nanochains network depending on the number of depositions (see Figure $1(b)$ as an example of the nanochain network formed after a single deposition). The electrical conductivity has been calculated by estimating the cross-sectional area of the nanochains from a number of AFM images and known dimensions of the electrode geometry. The conductivity of the Ni nanochain network was calculated to be $0.0024 \mathrm{~S} /$ $\mathrm{m}$ after four depositions, which is relatively low considering the number density of the nanochains formed for each deposition. The high electrical resistance of the nanochains observed here is possibly due to the high interparticle resistance in the chain. Formation of $\mathrm{NiO}$ skin layer on top of Ni nanoparticles should be expected during their preparation process. $\mathrm{NiO}$ is known to be a good insulator with the conductivity significantly lower than that for $\mathrm{Ni}\left(10^{-10}-10^{-}\right.$ ${ }^{5} \mathrm{~S} / \mathrm{m}$ ) depending on the grain size and preparation con- dition [42]. Therefore based on the measured conductivity values we can assume small degree of nanoparticles' surface oxidation.

Magnetic properties of the nanoparticles and the nanochains were studied by employing two prime techniques. First, magnetic force microscopy (MFM) which involves the sequential mapping of the topography in tapping mode (tip-sample separation of $\sim 10 \mathrm{~nm}$ ) and the stray magnetic field (tip-sample separation of $\sim 50 \mathrm{~nm}$ ) using a magnetized tip has been employed to obtain a high resolution map of the magnetic stray field of the nanochain sample [43]. Second, magnetic properties as a function of temperature and magnetic field strength were studied with the help of a Superconducting Interference Device (SQUID, Quantum Design MPMS XL-7) magnetometer.

Figure 3(a) depicts the topography and Figure 3(b) shows the corresponding MFM image on a Ni nanochain sample. While the topography image demonstrates a granular nature of the chains, the MFM image reveals variation 
of the dipolar magnetic stray field along the chains with the most probable arrangement of magnetic moments perpendicular to the chain line. Contrast observed in the MFM image is a result of interaction of magnetic moments on the sample surface with the magnetic moment of the AFM tip (which has a direction normal to the surface plane). The radius of the curvature of the tip is comparable to the size of nanoparticles in our case. In cases of head-to-tail "ferromagnetic type" (Figure 3(c)) arrangement of the magnetic moments within a nanochain, an attraction-repulsion interaction between the tip and the nanoparticles would reproduce exactly their topography image (higher interaction near nanoparticle centers and weaker interactions near inter-particle boundaries). However, in cases of antiparallel "antiferromagnetic type" arrangement of nanoparticles' magnetic moments along the chain, the situation is more complex. Because of relatively long-range forces of magnetic interactions, the AFM tip "feels" neighboring particles (with orientation of the local magnetic field opposite to the magnetic field of the particle being probed at a given point of time). This multi-particle interaction (at least two particles of the chain and one of the AFM tip) manifests itself as an appearance of a "granular" structure (Figure 3(b)), which is significantly different from AFM topography image (Figure 3(a)). The strength of magnetic interaction between the tip and a nanoparticle is higher when the tip is located out of the center of particular particle (due to interaction with the neighboring particles) but weaker near the center and on the boundaries. In the suggested model (Figure 3(c)), each nanoparticle represents one small ferromagnetic domain (i.e. a domain with uniform orientation of the magnetic field of the magnetic moment). Orientation of the magnetic moments of individual nanoparticles within a chain is antiparallel, which produces a net antiferromagnetic order. The chain can be considered as 1D antiferromagnetically ordered state as evidenced subsequently. Figure 3(d) represents a "zoom-in" portion of the MFM image presented in Figure 3(b) with application of Fourier filter to cut off the high frequency noise. One can see that each $\mathrm{Ni}$ nanoparticle chain is represented by a double string line with alternation of the magnetic field strength along the strings, which is in accordance to the antiparallel arrangement of the magnetic moments (perpendicular to the nanoparticle chain) presented in the Figure 3(c). Ferromagnetic head to tail arrangement of the magnetic moment of individual particles within the chain (Figure 3(c)) would produce a single string of magnetic field distribution for each nanoparticle chain, which is not observed in our case. Smaller details of the magnetic field distribution cannot be seen because of limitations of the MFM technique resolution.

Figure 4 shows the temperature dependence of the magnetic moment of nanochains on glass substrate. The measurement is initialized by zero-field cooling (ZFC) the sample down to $T=5 \mathrm{~K}$. The lower branch depicts the subsequently recorded field-heating data which are obtained on heating the sample from $T=5$ to $380 \mathrm{~K}$ in a planar applied magnetic field of $\mu_{0} H=50 \mathrm{mT}$. The upper branch shows the field-cooling (FC) data obtained on subsequent cooling in the presence of the magnetic field down to $T=5 \mathrm{~K}$.

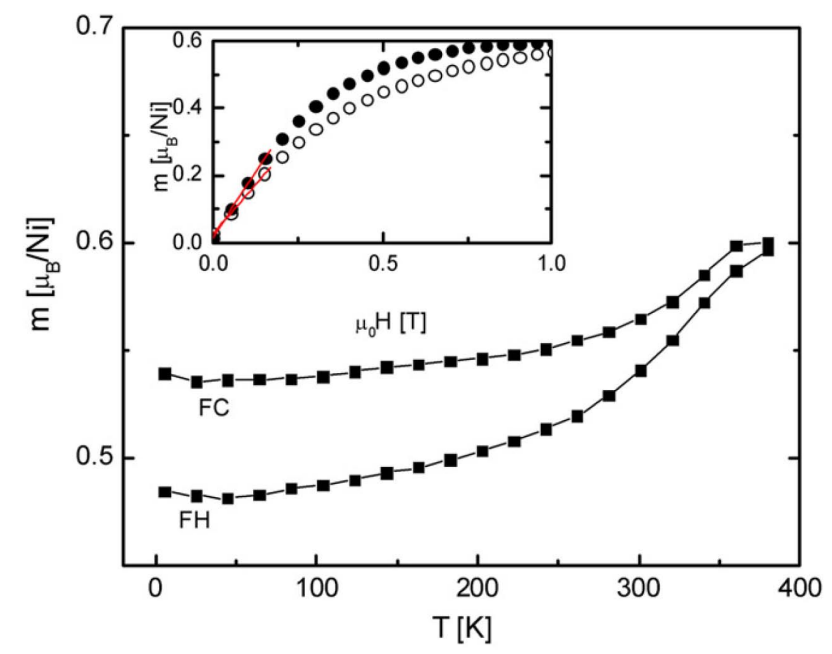

Figure 4. Temperature dependence of $m$ versus $T$ curves of the nanochain sample. Measurement is initialized by a zero-fieldcooling (ZFC) procedure. Arrow to the right indicates $m$ versus $T$ data for subsequent field heating in $\mu_{0} H=50 \mathrm{mT}$. Arrow to the left marks the field-cooling (FC) branch. The inset shows two virgin $m$ versus $\mu_{0} H$ isotherms for $T=5 \mathrm{~K}$ (open circles) and $T=380$ $\mathrm{K}$ (solid circles), respectively. The lines are best fits to the first four data points, respectively. The slopes determine the corresponding susceptibilities, which allow for comparison with the $m$ versus $T$ data (see text).

ZFC and FC branches exhibit irreversibility commonly encountered in superparamagnetic blocking behavior of nanoparticles. Furthermore, the observation of decrease of FC magnetization with decreasing temperature has often been regarded as an indication of collective super-spin-glass state [18]. We cannot exclude this possibility here and this issue remains to be addressed in the future studies. However, careful inspection of the data reveals a clear signature of AF order in agreement with the MFM results (see Figure $3(b))$, which suggests AF order along the chains. Note, that this type of order can only be short range since there is no finite temperature phase transition in 1D systems.

Collective magnetic states are experimentally well known in nanoparticle ensembles although the origin of the interaction is often unclear [44-48]. Here, a first fingerprint of $\mathrm{AF}$ order in the nanochain sample is associated with the FC branch of the magnetic moment $m$ versus $T$ data. While superparamagnetic blocking of monodisperse particles results in a temperature independent FC curve [49], and polydispersity of independent nanoparticles even gives rise to increasing FC magnetization with decreasing temperature [50], here, the AF order along the chains results in a significant decrease of magnetization with deceasing temperature.

The field induced offset moment $m\left(T=5 \mathrm{~K}, \mu_{0} H=50\right.$ $\mathrm{mT})=3.1 \times 10^{-8} \mathrm{~A} \mathrm{~m}^{2}$ of the ZFC curve resembles the topological disorder of the nanochains (see Figure 4). The latter gives rise to a statistical mixture of parallel and perpendicular AF susceptibility contributions according to $\chi_{\text {random }}=$ $1 / 3 x_{\|}+2 / 3 x_{\perp}$ [51]. Following mean-field arguments, the perpendicular susceptibility, $\chi_{\perp}$ versus $T$, is roughly constant for temperatures below the Néel temperature, $T_{N^{\prime}}$ while the parallel susceptibility, $x_{\|}$versus $T$, levels off to 
zero for $T \rightarrow 0$. Therefore, we can expect a statistical mixture of these two susceptibility contributions that yields $\chi_{\text {random }}(T=5 \mathrm{~K}) / \chi_{\text {random }}(T=380 \mathrm{~K}) \approx 2 / 3$, assuming that $\chi_{\|}(T$ $=5 \mathrm{~K}) \approx 0$, and $\chi_{\perp}(T=380 \mathrm{~K}) \approx \chi_{\perp}\left(T=T_{\mathrm{N}}\right)$. In fact, the data of the ZFC branch reveal $\chi_{\text {random }}(T=5 \mathrm{~K}) / \chi_{\text {random }}(T=380 \mathrm{~K})=$ 0.81 which is within an error of $\sim 20 \%$, in reasonable agreement with the rough mean-field estimation. Subsequently, we rule out that this susceptibility ratio and the equivalent magnetization ratio resembles simple superparamagnetic blocking scenario. In order to show the impact of interaction, we estimate the ratio expected in the framework of the Néel Brown model for superparamagnetic non-interacting nanoparticles. In the latter case, after ZFC the field induced magnetization in the blocked low temperature limit reads $M_{\mathrm{ZFC}}^{\mathrm{bP}}=2 \varepsilon M_{\mathrm{s}} h / 3$, where $\varepsilon$ is the volume fraction of the sample occupied by the FM nanoparticles, $M_{\mathrm{s}}$ is the nanoparticle saturation magnetization and $h$ is the normalized magnetic field. Here, normalization refers to the coercive field of a particle where coherent magnetization reversal according to Stoner-Wohlfarth behavior is assumed [52]. The magnetization at the blocking temperature, $T_{\mathrm{B}^{\prime}}$ is in turn given by $M_{\mathrm{FC}}^{\mathrm{bl}}=\varepsilon M_{\mathrm{s}} L\left(2 \ln \left(\tau_{m} / t_{0}\right) /(1-h)^{2}\right)$, which is constant for $T<T_{B}$ in the Néel Brown model for monodisperse particles. Here $L(x)$ is the Langevin function, $\tau_{m} \approx 1 \mathrm{~s}$ is the characteristic timescale for a DC SQUID measurement and $\tau_{0} \approx 10^{-9} \mathrm{~s}$ is the inverse attempt frequency quantifying the rate at which a thermally activated nanoparticle attempts to overcome the energy barrier which separates the up from the down magnetized state [52]. With $\tau_{m}$ and $\tau_{0}$ from above

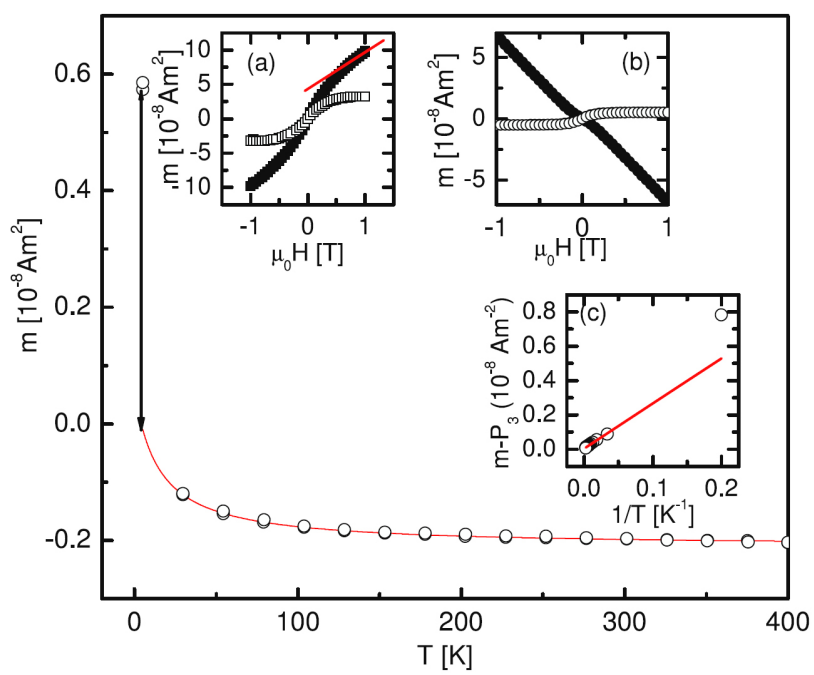

Figure 5. Temperature dependence of $m$ versus $T$ curves of the magnetic moment of the nanoparticle sample. ZFC and FC ramps are measured in an applied planar field of $\mu_{0} H=50 \mathrm{mT}$ after zerofield cooling. Inset (a) shows the isothermal $m$ versus $\mu_{0} H$ hysteresis for $T=5 \mathrm{~K}$. Solid squares are raw data including a linear background contribution, which corresponds to the excess moment determined by the fit. Open squares show the data after background subtraction. Inset (b) show the $m$ versus $\mu_{0} H$ hysteresis for $T=380 \mathrm{~K}$ before (solid circles) and after subtraction (open circles) of the diamagnetic background. Inset (c) shows $m-P_{3}$ versus $1 / T$, indicating superparamagnetic type Curie-Weiss behavior of a second fraction of nanoparticles. and the approximation $L(x) \approx x / 3$ we obtain $M_{\mathrm{ZFC}}^{\mathrm{bl}} / M_{\mathrm{FC}}^{\mathrm{bl}}(T$ $\left.=T_{\mathrm{B}}\right) \approx 1 / 21 \approx 0.05$, resembling a much larger dynamics of the ZFC $m$ versus $T$ curve than the actual data show in Figure 4 in accordance with $\chi_{\text {random }}(T=5 \mathrm{~K}) / \chi_{\text {random }}(T=380 \mathrm{~K})$ $=0.81$. Note that the linearization of the Langevin function holds for small magnetic fields of the isotherms $m$ versus $H$ where the ratio of the magnetization values at $T=5$ and $380 \mathrm{~K}$ and the susceptibility ratios at these temperatures are identical. The inset of Figure 4 shows that this linear approximation is justified for the applied fields $\mu_{0} H=50 \mathrm{mT}$, which is small in comparison to the saturation field.

The inset of Figure 4 shows two virgin $m$ versus $\mu_{0} H$ curves corresponding to full hysteresis loops (not shown) of the nanochain sample for $T=5 \mathrm{~K}$ (open circles) and $T$ $=380 \mathrm{~K}$ (full circles), respectively. At first glance it is surprising that the high temperature isotherm $m$ versus $\mu_{0} H$ is steeper than that at $T=5 \mathrm{~K}$. The data are, however, in perfect agreement with the $m$ versus $T$ behavior and their interpretation as a signature of AF order. In order to evidence this consistency we compare the susceptibility ratio $\chi_{\text {random }}(T=5 \mathrm{~K}) / \chi_{\text {random }}(T=380 \mathrm{~K})=0.81$, obtained from the $m$ versus $T$ data with the ratio of the initial slopes $\mathrm{d} m$ / $\mathrm{d}\left(\mu_{0} H\right)$ of the corresponding isotherms. The susceptibilities are expected to be proportional to the slopes $\mathrm{d} m / \mathrm{d}\left(\mu_{0} H\right)$ of $m$ versus $H$ at $T=5$ and $380 \mathrm{~K}$, respectively. The latter are determined from linear best fits (lines in the inset) of the first four data points of the isotherms, respectively. Their ratio is given by $\mathrm{d} m(T=5) / \mathrm{d}\left(\mu_{0} H\right) / \mathrm{d} m(T=380 \mathrm{~K}) / \mathrm{d}\left(\mu_{0} H\right)$ $=0.76$, in close agreement with the ratio of the susceptibilities determined from $m$ versus $T$ data. The increasing slope of $m$ versus $\mu_{0} H$ with increasing temperature reflects the decrease of AF order, which results in increasing field induced magnetization.

Figure 5 shows magnetization measurements performed on isolated nanoparticles cast on substrates in the absence of magnetic field. The ZFC and FC $m$ versus $T$ curves were measured for an applied field of $\mu_{0} H=50 \mathrm{mT}$ after zerofield cooling from $400 \mathrm{~K}$ down to $T=5 \mathrm{~K}$. In contrast to the nanochains system, no splitting between the heating and the cooling ramp was observed. This sample behaves qualitatively like a conventional non-blocked paramagnet with a strong diamagnetic background in the temperature interval $30 \mathrm{~K}<\mathrm{T}<400 \mathrm{~K}$. However, at low temperatures $T<10$ $\mathrm{K}$ the magnetic moment strongly increases and a deviation from simple paramagnetism sets in.

This becomes obvious when fitting $m$ versus $T$ for $30 \mathrm{~K}$ $\leq T \leq 400 \mathrm{~K}$ to the Curie-Weiss type function $m=P_{1} /(T$ - $\left.P_{2}\right)+P_{3}$, where $P_{1}, P_{2}$, and $P_{3}$ are various fit parameters. This ansatz takes into account the diamagnetic background of the glass substrate via $P_{3}$ and allows for deviations from simple paramagnetic behavior via $P_{2}$. While $P_{2}>$ 0 expresses FM order, $P_{2}<0$ indicates antiferromagnetism. The fit yields $P_{1}=3.96 \times 10^{-8} \pm 1.7 \times 10^{-9} \mathrm{~A} \mathrm{~m}^{2} \mathrm{~K}, P_{2}=-14.7$ $\pm 1.8 \mathrm{~K}$ and a diamagnetic background at $\mu_{0} \mathrm{H}=50 \mathrm{mT}$ of $P_{3}$ $=-2.1 \times 10^{-9} \pm 7 \times 10^{-12} \mathrm{~A} \mathrm{~m}^{2}$. Surprisingly, also the larger separated nanoparticles have a tendency towards AF order indicated by the negative Curie-Weiss temperature. In the nanoparticle system however, AF interaction is much weaker than in the nanochain system. Remarkably, the ex- 
trapolation of the fit towards $T=5 \mathrm{~K}$ reveals a significant deviation from the measured data point $m\left(T=5 \mathrm{~K}, \mu_{0} H=\right.$ $50 \mathrm{mT}$ ). This deviation indicates the presence of a second group of nanoparticles which behaves essentially as a dominant paramagnetic background when $m$ versus $\mu_{0} H$ is considered at $T=5 \mathrm{~K}$. The $m$ versus $\mu_{0} H$ isotherm at $T=5 \mathrm{~K}$ is shown in the inset (a) of Figure 5. The solid squares exhibit a superposition of a saturating hysteresis loop and a background signal with linear field dependence. The slope $\mathrm{d} m$ / $\mathrm{d}\left(\mu_{0} H\right)=6.5 \times 10^{-8} \mathrm{~A} \mathrm{~m}^{2} / \mathrm{T}$ of $m(T=5 \mathrm{~K})$ versus $\mu_{0} H$ is determined from a linear fit to the data points at $0.85 \mathrm{~T} \leq \mu_{0} \mathrm{H}$ $\leq 1 \mathrm{~T}$. It is in reasonable agreement with the excess susceptibility $\tilde{\chi}_{\mathrm{ex}}=1.2 \times 10^{-7} \mathrm{~A} \mathrm{~m}^{2} \mathrm{~T}^{-1}$, which corresponds to the excess moment $m_{\mathrm{ex}}=5.9 \times 10^{-9} \mathrm{~A} \mathrm{~m}^{2}$, measured at $\mu_{0} H=50$ $\mathrm{mT}$ and displayed in Figure 5 (arrow).

Similarly, inset (b) shows the isotherm $m$ versus $\mu_{0} \mathrm{H}$ at $T=380 \mathrm{~K}$. The raw data (solid circles) are dominated by a large diamagnetic background, which originates from the glass substrate. Again the background of the isotherm is in qualitative agreement with $P_{3}=-2.1 \times 10^{-9} \pm 7 \times 10^{-12} \mathrm{~A} \mathrm{~m}^{2}$ determined from the $m$ versus $T$ data at $\mu_{0} H \approx 50 \mathrm{mT}$, which predicts a diamagnetic susceptibility of $\tilde{X}_{\text {dia }}=-4.2 \times 10^{-8} \mathrm{~A}$ $\mathrm{m}^{2} \mathrm{~T}^{-1}$. A linear fit of $m$ versus $\mu_{0} \mathrm{H}$ at $T=380 \mathrm{~K}$ yields $\mathrm{d} m /$ $\mathrm{d}\left(\mu_{0} H\right)=-7.3 \times 10^{-8} \mathrm{~A} \mathrm{~m}^{2} \mathrm{~T}^{-1}$.

To quantify the fraction of superparamagnetic contribution we plot $\left(m-P_{3}\right)$ versus $1 /\left(T-P_{2}\right)$ in Figure 5(c). Fit of the data to Curie-Weiss (CW) law yields $\mathrm{CW}$ the slope $=$ $0.38 \times 10^{-8} \mathrm{~A} \mathrm{~m}^{-2} \mathrm{~K}^{-1}$. The deviation of the $\mathrm{T}=5 \mathrm{~K}$ data from the Curie-Weiss fit is $0.25 \times 10^{-8} \mathrm{~A} \mathrm{~m}^{-2} \mathrm{~K}^{-1}$.

In Figure 6 we compare the hysteresis loops of the nanochain sample (solid circles) and the nanoparticle sample (open circles), measured at $T=5 \mathrm{~K}$. The magnetic moment is normalized to the value $m\left(\mu_{0} H=1 \mathrm{~T}\right)$, respectively. Clearly, the loop for the nanoparticles is virtually free from hysteresis in accordance with non-blocked paramagnetic behavior. The nanochain sample reveals, however, clear hysteretic behavior. Alternation of the magnetic filed along each of the individual chains (one-dimensional antiferromagnetic structure) is also evident from the MFM image (Figure 3(b)). Such type of antiparallel arrangement of dipole moments during self- assembly process arrangement has previously been predicted for chains of nanoparticles and magnetic nanosystems [53].

In summary, we have demonstrated a rather simple physical method for the fabrication of monodisperse magnetic nanoparticles. Due to local coupling between nanosized magnetic moments, we find that Ni particles of diameter $9.5 \mathrm{~nm}$ organize themselves into long chains when subjected to external magnetic field. Furthermore, preferential alignment of the nanochains was achieved by combing the external magnetic field with template assisted assembly and flow field. The self-assembled nanochains exhibited rather unusual nano-magnetic properties. 1D antiferromagnetic order (antiparallel arrangement of magnetic moments with magnetization direction perpendicular to the long axis of chains) is evidenced by magnetic force microscopy and SQUID magnetometry. The field-heating magnetization data has been interpreted by a random mixture of parallel and perpendicular antiferromagnetic

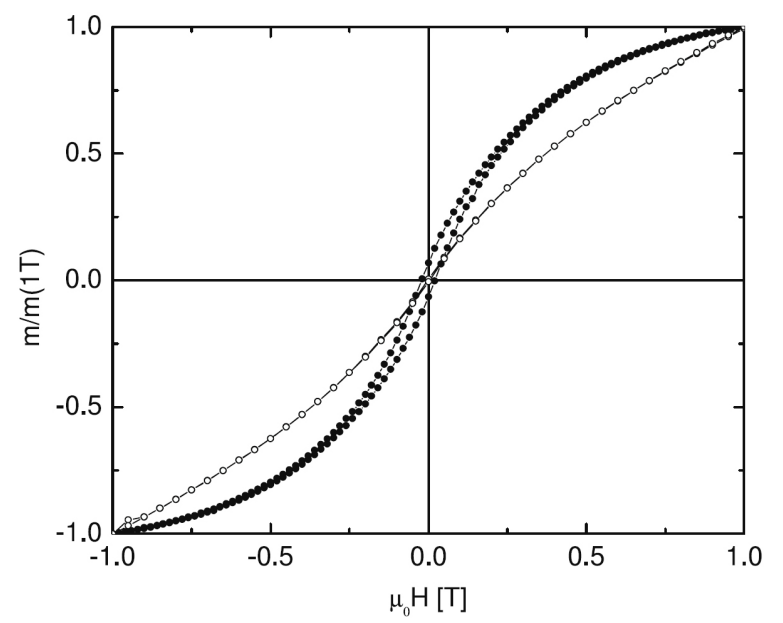

Figure 6. Hysteresis loops $m$ versus $\mu_{0} H$ at $T=5 \mathrm{~K}$ of the nanochain sample (solid circles) and the nanoparticle sample (open circles), respectively. Both curves are normalized with respect to the magnetic moment at $\mu_{0} H=1 \mathrm{~T}$.

susceptibility contributions. Ruling out simple superparamagnetic blocking behavior, splitting between the ZFC and FC magnetization branches is attributed to random field effects known from diluted antiferromagnets in a field. In contrast to the complex magnetic behavior of the Ni nanochains, the isolated Ni nanoparticles exhibited a paramagnetic behavior. A bimodal decay into distinct nanoparticle species is corroborated by low temperature magnetometry. Magneto-resistance properties the Ni nanochains network, which exhibits a macroscopic electrical percolation, would be interesting for potential sensor applications.

\section{Acknowledgments}

We gratefully acknowledge support by NSF through Career DMR-0547887 and MRSEC DMR-0213808 at the University of Nebraska.

\section{References}

[1] Bader S D 2006 Rev. Mod. Phys. 78 1-15

[2] Chou S Y 1997 Proc. IEEE 85 652-71

[3] Parkin S S P et al 1999 J. Appl. Phys. 85 5828-33

[4] Prinz G A 1998 Science 282 1660-3

[5] Martin J I, V'elez M, Moralesa R, Alameda J M, Anguitab J V, Briones F and Vicent J L 2002 J. Magn. Magn. Mater. 249 156-62

[6] Binek Ch and Doudin B 2005 J. Phys.: Condens. Matter 17 L39-44

[7] Kronick P and Gilpin R W 1986 J. Biochem. Biophys. Methods 12 73-80

[8] Gupta P K and Hung C T 1989 Life Sci. 44 175-86

[9] Ross C A 2001 Annu. Rev. Mater. Res. 31 203-35

[10] Wolf S A, Awschalom D D, Buhrman R A, Daughton J M, von Molnár S, Roukes M L, Chtchelkanova A Y and Treger D M 2001 Science 294 1488-95

[11] Skomski R, Chipara M and Sellmyer D J 2003 J. Appl. Phys. 93 7604-6 
[12] Chappert C, Fert A and Nguyen Van Dau F 2007 Nat. Mater. 6 813-23

[13] Chou S Y, Krausss P R and Kong L J 1996 J. Appl. Phys. 79 6101-6

[14] O'Grady K and Laidler H J 1999 J. Magn. Magn. Mater. 200 616-33

[15] Dormann J L, Cherkaoui R, Spinu L, Nogues M, Lucari F, D'Orazio F, Fiorani D, Garcia A, Tronc E and Jolivet J P 1998 J. Magn. Magn. Mater. 187 L139-44

[16] Djurberg C, Svedlindh P, Nordblad P, Hansen M F, Bodker F and Morup S 1997 Phys. Rev. Lett. 79 5154-7

[17] Mamiya H, Nakatani I and Furubayashi T 1999 Phys.Rev.Lett. 82 4332-5

[18] Jönsson P E 2004 Adv. Chem. Phys. 128 191-248

[19] Green M 2005 Chem. Commun. 24 3002-11

[20] Cheng G J, Puntes V F and Guo T 2006 J. Colloid Interface Sci. 293 430-6

[21] Hou Y, Kondoh H, Ohta T and Gao S 2005 Appl. Surf. Sci. 241 218-22

[22] Cordente N, Amiens C, Chaudret B, Respaud M, Senocq F and Casanove M J 2003 J. Appl. Phys. 94 6358-65

[23] Sun S, Murray C B and Doyle H 1999 Advanced hard and soft magnetic materials Mater. Res. Soc. Symp. Proc. 577385

[24] Puntes V F, Krishnan K M and Alivisatos A P 2001 Science 291 2115-7

[25] Murray C B, Kagan C R and Bawendi M G 1995 Science 270 1335-8

[26] Murray C B, Sun S, Doyle H and Betley T 2001 MRS Bull. 26 985-91

[27] Ozin G A 1992 Adv. Mater. 4 612-49

[28] Bentzon M D, van Wonterghem J, Mørup S and Thölén A 1989 Phil. Mag. B 60 169-78

[29] Zhou W L, Carpenter E E, Lin J, Kumbhar A, Sims J and O'Connor C J 2001 Eur. Phys. J. D 16 289-92

[30] Lalatonne Y, Richardi J and Pilnei M P 2004 Nat. Mater. 3 121-5

[31] Li J, Ye Q, Cassell A, Ng H T, Stevens R, Han J and Meyyappan M 2003 Appl. Phys. Lett. 82 2491-3

[32] Pileni M P 2001 J. Phys. Chem. B 105 3358-71

[33] Whitney T M, Jiang J S, Searson P C and Chien C L 1993 Science 261 1316-19
[34] Kirschvink J L, Walker M M and Diebel C E 2001 Curr. Opin. Neurobiol. 11 462-7

[35] Singamaneni S and Bliznyuk V 2005 Appl. Phys. Lett. 87 162511

[36] Favier F, Walter E, Zach M P, Benter T and Penner R M 2001 Science 293 2227-31

[37] Atashbar M Z, Banerji D, Singamaneni S and Bliznyuk V 2004 Nanotechnology 15 374-8

[38] Atashbar M Z, Banerji D and Singamaneni S 2005 IEEE Sensors J. 5 792-7

[39] Chen J and Weimer W A 2002 J. Am. Chem. Soc. 124 758-9

[40] Bensimon A, Simon A, Chiffaudel A, Croquette V, Heslot F and Bensimon D 1994 Science 265 2096-8

[41] Ko H, Peleshanko S and Tsukruk V V 2004 J. Phys. Chem. 108 4385-93

[42] Biju V and Abdul Khadar M 2001 Mater. Res. Bull. 36 21-33

[43] Kaiser U, Schwarz A and Wiesendanger R 2007 Nature 446 522-5

[44] Kleemann W, Petracic O, Binek Ch, Kakazei G N, Pogorelov Yu G, Sousa J B, Cardoso S and Freitas P P 2001 Phys. Rev. B 63134423

[45] Sahoo S, Petracic O, Kleemann W, Nordblad P, Cardoso S and Freitas P P 2003 Phys. Rev. B 67214422

[46] Chen X, Sichelschmidt O, Kleemann W, Petracic O, Binek Ch, Sousa J B, Cardoso S and Freitas P P 2002 Phys. Rev. Lett. 89 137203

[47] Puntes V F, Gorostiza P, Aruguete D M, Bastus N G and Alivisatos A P 2004 Nat. Mater. 3 263-8

[48] Salgueiriño-Maceira V, Correa-Duarte M A, Bañobre-López M, Grzelczak M, Farle M, Liz-Marz'an L M and Rivas J 2008 Adv. Funct. Mater. 991-6

[49] Han M, Liu Q, He J, Song Y, Xu Z and Zhu J 2007 Adv. Mater. 191096

[50] Fonseca F C, Goya G F, Jardim R F, Muccillo R, Carreno N L V, Longo E and Leite R 2002 Phys. Rev. B 66104406

[51] Kittel C 1996 Introduction to Solid State Physics 7th edn (Hoboken, NJ: Wiley)

[52] Bitoh T, Ohba K, Takamatsu M, Shirane T and Chikazawa S 1995 J. Phys. Soc. Japan 64 1305-10

[53] Tlusty T and Safran S A 2000 Science 290 1328-31 